Egyptian

Orthodontic Journal

\title{
EVALUATION OF EARLY ANTIMICROBIAL EFFECT OF ORTHODONTIC ADHESIVES AND CEMENTS
}

\author{
Fatma H Ghaith*, Ahmad A Ramadan**, \\ Hassan N El-Islam***, Nabeel E Abu Shamaa****.
} ABSTRACT:

Introduction: Patients undergoing fixed appliance treatment are at greater risk for increases in salivary and plaque levels of Streptococcus mutans and an elevated risk of dental caries. The purpose of this study was to investigate if the commercially available orthodontic adhesive and cements would have antimicrobial properties. Methods: Six dental materials were evaluated for antimicrobial properties: a chemically cured composite with fluoride (Reliance), a light cured composite with fluoride (Reliance light bond), a chemically cured composite without fluoride (Monolok 2 RMC), a light cure composite without fluoride (alpha dent Lincolnwood), a glassionomer cement (Vivaglass CEM PL), and a polycarboxylate cement (Alpha-Dent). Streptococcus mutans strain was grown aerobically in brain heart infusion (BHI) broth containing $0.5 \%$ bacitracin at $37^{\circ} \mathrm{C}$ for 48 hrs. Under sterile conditions, these materials were placed onto plates of mitis salivarius agar and agar diffusion test (ADT) was performed. Results: the results indicated a visible zone of inhibition around the specimens which were measured and analyzed statistically by $t$ and ANOVA tests. GIC (Vivaglass CEM PL), polycaboxylate cement and chemically activated composite containing fluoride (Reliance Orthodontic Products Inc)

\footnotetext{
* Master Degree Student

** Professor and Head, Orthodontic Department, Faculty of Dentistry, Suez Canal University

*** Professor and Head, Microbiology Department, Faculty of Medicine, Suez Canal University.

${ }^{* * * *}$ Lecturer, Orthodontic Department, Faculty of Dentistry, Suez Canal University.
} 
Egyptian

Orthodontic Journal

were significant at 5\% level of confidence. GIC cement was the highest material exhibiting antimicrobial potency.

Conclusion: The use of glass ionomer cement (Vivaglass CEM PL) for orthodontic bands cementation and a chemicallycured composite resin with fluoride (Reliance Orthodontic Prod Inc.) for bonding brackets reduced early activity of $S$. mutans bacterial strain. Also, fluoride content in the orthodontic adhesives and cements had an effect in reducing early activity of S. mutans.

Key words: Agar Diffusion test ADT, Brain Heart Infusion (BHI) Groth, Streptococcus mutans, Orthodontic adhesives and cements, Antimicrobial property.

\section{INTRODUCTION}

Fixed orthodontic appliances are significant challenge to the patient in regard to maintaining good oral hygiene and avoiding or minimizing decalcification of enamel during orthodontic treatment. ${ }^{1-3}$ The bands or brackets and the various orthodontic elements (elastics, plastic sleeves, springs, cleats, buttons, and archwires) render oral hygiene more difficult and increase plaque accumulation ${ }^{4,5}$ especially in the maxillary incisor area. ${ }^{6}$ The increase of oral colonization by Streptococcus mutans which in turn increases the risk for the development of dental caries. ${ }^{7}$ Caries development in the form of white spot lesions on the labial surfaces of the teeth, particularly maxillary incisors, is an esthetic side effect of treatment with fixed orthodontic appliances. $^{8}$

The practice of orthodontics is constantly improving with the use of new techniques and materials that benefit both the patient and the clinician. As a result, various attempts have been made to minimize white spot lesion formation during orthodontic treatment. ${ }^{8-11}$ In a study by $\emptyset$ gaard et $\mathrm{al}^{8}$ they found that with the use of a fluoride varnish in combination with and without the use of a chlorhexidine varnish, there was a significant reduction in the incidence of white spot lesion formation particularly in the maxillary incisor region. Buyukyilmaz and $\emptyset_{\text {gaard }^{9}}$ in an earlier study suggested the use of antimicrobials in combination with fluorides to improve the cariostatic effect of fluoride.

Composite resins and glass ionomer cements (GICs) are widely used in securing fixed orthodontic devices. During the 1990s, a major development of

Volume 33 - June 2008 
hybridization technology underlying composite resins and GICs popularized the use of resin-modified GICs (RMGICs) and polyacid-modified composites (compomers) in orthodontics.

Composites used as orthodontic direct bonding adhesives have a polymeric matrix that can host and nurture a variety of aerobic and anaerobic micro-organisms acting alone or in combination (consortia). Their accumulation can lead to premature debonding and eventually enamel decalcification and periodontal diseases. ${ }^{12}$ The use of fluoride- containing orthodontic bonding materials both in vitro ${ }^{13,14}$ and in vivo ${ }^{15}$ has shown variable results in helping to prevent or reduce decalcification. Significantly elevated levels of fluoride in plaque have been found adjacent to GIC-retained brackets compared with those adjacent to composite resin-retained brackets up to 6 months after the onset of treatment ${ }^{16}$.

The aim of this in vitro study was to investigate the antimicrobial characteristics exhibited by six commonly used orthodontic materials ( 4 adhesives and 2 cements), using the Agar Diffusion Test (ADT) on one of the initial colonizers of the oral biofilm architecture, Streptococcus mutans. The orthodontic adhesive and cement materials-bacteria interaction was evaluated in fresh specimens.

\section{Materials and Methods:}

\section{Adhesives and Cements Evaluated and Grouping:}

The bonding agents and cements used in this study were classified into 6 groups (Table 1).

Table 1: Orthodontic materials (adhesives and cements) used in this study.

\begin{tabular}{|c|c|c|c|}
\hline Group & Material & Composition & Manufacturer \\
\hline $\mathbf{A}$ & $\begin{array}{l}\text { Chemically-cured } \\
\text { composite resin }\end{array}$ & Contains fluoride & $\begin{array}{l}\text { Reliance Orthodontic Products Inc., } \\
\text { Itasca, IL }\end{array}$ \\
\hline B & $\begin{array}{l}\text { light-cured composite } \\
\text { resin }\end{array}$ & Contains fluoride & Reliance Ortho Prod, Light bond ${ }^{\mathrm{TM}}$ \\
\hline $\mathbf{C}$ & $\begin{array}{l}\text { Chemically-cured } \\
\text { composite resin }\end{array}$ & $\begin{array}{l}\text { Does not contain } \\
\text { fluoride }\end{array}$ & Monolok 2 RMC, Inc USA \\
\hline D & $\begin{array}{l}\text { Light-cured composite } \\
\text { resin }\end{array}$ & $\begin{array}{l}\text { Does not contain } \\
\text { fluoride }\end{array}$ & alpha dent Lincolnwood Illinois, \\
\hline $\mathbf{E}$ & Glass ionomer cement & Contains fluoride & $\begin{array}{l}\text { Vivaglass CEM PL, ivoclar vivadent } \\
\text { clinical, Schannn / Liechtenstein }\end{array}$ \\
\hline $\mathbf{F}$ & Polycarboxylate cement & $\begin{array}{l}\text { Does not contain } \\
\text { fluoride }\end{array}$ & Alpha-Dent, Illinois USA \\
\hline
\end{tabular}


Egyptian

Orthodontic Journal

\section{Bacterial strain and media:}

Streptococcus mutans strain was used in all the experiments and obtained from Microbiology Department, Faculty of Veterinary Medicine, Cairo University. S mutans was grown aerobically from frozen stock cultures in brain heart infusion (BHI) broth containing $0.5 \%$ bacitracin at $37^{\circ} \mathrm{C}$ for $48 \mathrm{hrs}$. Bacteria were used at their late-logarithmic to early-stationary phase.

Mitis Salivarius Agar [Difco, USA] was used as it is the selective medium specific for the growth of Streptococcus mutans bacteria. It is characterized by its blue color giving a characteristic form upon growth of Streptococcus mutans (small black colonies).

\section{Preparation of Mitis Salivrius Agar medium:}

The preparation of this medium was done as follows:

1- Eighteen grams of (Mitis Salivrius) powder were suspended in $200 \mathrm{ml}$ of double diluted purified water.

2- Thoroughly mixing was done during heating with frequent agitation and boiling for 1 minute to completely powder dissolving.

3- Then, autoclaving at $121^{\circ} \mathrm{C}$ for 15 minutes.

4- The medium was then left to cool down to reach about $50-55^{\circ} \mathrm{C}$.

5- Two hundred $\mu \mathrm{l}$ of chapman (tellurite-k) solution were added using sterilized plastic Petri dishes, (4 mm in thickness) and then left until complete cooling. This amount were enough for 15 plates (dishes) of diameter $90 \mathrm{~mm}$.

\section{Bonding and cement materials:}

All experimental specimens were made following the manufacturers' instructions. Groups B and D were light activated, while groups A and C were allowed to bench cure. Group E and group F were allowed to set.

In the present study Agar Diffusion Test (ADT) ${ }^{17}$ was used to evaluate the interaction of orthodontic adhesives and cements with the bacteria.

\section{The Agar Diffusion Test:}

1- Each plate was inoculated with $200 \mu 1$ of freshly grown S. mutans after measuring the optic density $\left(\mathrm{OD}_{650}\right)$ of the bacterial broth culture [Brain Heart Infusion (BHI) broth]. As it is a liquid medium for the growth of Streptococcus mutans 
2- The broth containing aerobically grown bacteria S. mutans was adjusted to obtain OD 0.6 at $650 \mathrm{~nm}$ by using the spectrophotometer [JENWAY, Germany], in order to have $0.9-1.1 \times 10^{6}$ colony forming units (CFU) in 10 $\mu \mathrm{l}$ bacterial suspension.

3- $2.5 \mu \mathrm{l}$ of the bacterial broth by micropiptte were inserted in the cuovett and after the dilution of the broth was adjusted to reach the 0.6 concentrate of bacteria in broth.

4- The freshly grown S. mutans was spreaded by swab on the agar plate surface.

5- Six holes, $4 \mathrm{~mm}$ in diameter, were punched on the agar surface of each plate.

6- Then, the respective orthodontic adhesives and cements were introduced and polymerized immediately.

7- The plates were incubated at $37^{\circ} \mathrm{C}$ for 48 hours and then visually inspected for the presence of inhibition zones in bacterial lawn.

8- The bacterial inhibition zones halo were measured in two perpendicular locations and the mean expressed in millimeters.

9- The ADT for each material was repeated 4 times.

The specimens of each material was surrounded by halo zone in the bacterial lawn according to its antimicrobial effect and this zone was measured after 48 hours by two perpendicular locations and expressed in millimeters (Fig.1). The results are shown in table 2.

\section{Statistical analysis:}

Descriptive statistics including the mean, standard deviation, and minimum and maximum values were calculated for the six groups evaluated. Student's t-test and one way ANOVA test were used to compare the means of antimicrobial effect for the six materials. Significance was determined at the level of significance $\mathrm{P}<0.05$.

\section{RESULTS}

After ADT, the results indicated a visible zone of growth inhibition around the specimens of the tested materials. The highest inhibition zone was around group E, F,A,B,D and C respectively shown in table 2, graph 1.

Statistically by using $t$ and one way ANOVA tests revealed that GIC (Vivaglass CEM PL) was the highly significant material that had an antibacterial activity at 5\% level of confidence. Also, chemically activated composite resin was significant but of lower value, table 2 . 
Table 2, Graph 1: Illustrates the means ( $\mathrm{mm}$ ) and standard deviation of linear measurements of the halo inhibition zones of experimental groups.

\begin{tabular}{|c|c|c|c|c|}
\hline Group & Mean & Std. Deviation & Minimum & Maximum \\
\hline A & $7.600^{*}$ & .200 & 7.50 & 7.90 \\
\hline B & 7.225 & .263 & 7.00 & 7.50 \\
\hline C & 4.400 & .141 & 4.20 & 4.50 \\
\hline D & 4.425 & .096 & 4.30 & 4.50 \\
\hline E & $11.613^{*}$ & .193 & 11.40 & 11.80 \\
\hline F & $9.250^{*}$ & .957 & 8.00 & 10.00 \\
\hline
\end{tabular}

Group A: Chemical cure composite with fluoride.

Group C: Chemical cure composite without fluoride

Group B: Light cure composite with fluoride

Group E: Glass ionomer cement

Group D: Light cure composite without fluoride

Group F: Polycarboxylate cement $\quad * \mathrm{P}<0.05$

\section{DISCUSSION}

Most currently available orthodontic materials are not ideal in their clinical performance in terms of caries prevention. In the present study, 6 orthodontic materials were used for evaluating the antibacterial properties (4 adhesives and 2 cements). They were chosen to represent different categories of bonding and cementing orthodontic brackets and bands used in everyday orthodontic practice. It is accepted that by changing the microenvironment around bonded and cemented orthodontic devices, enamel demineralization can be better controlled. ${ }^{18,19}$ The specific bacterial strain (Streptococcus mutans) was selected for this study for its potential ability to affect white spot formation and thus dental caries. ${ }^{7,20,21} \mathrm{We}$ used agar diffusion test ADT as a quantitative method for evaluating the antibacterial properties by measuring the amount of halo zones (degree of bacterial inhibition) around the tested materials.

Conventional glass ionomer cements, which release and absorb fluoride have been explored as possible means of bracket bonding. Although their potential to prevent demineralization has been identified in laboratory studies, ${ }^{22}$ clinical trials have produced conflicting findings. ${ }^{23,24}$ In addition, these cements have unreliable and inferior adhesive properties compared to composite resins and, therefore, are not recommended for routine orthodontic bonding. ${ }^{25}$ So, we aimed in this study to test this material as cementing material for orthodontic bands.

Of the two band cements evaluated, GIC exhibited the highest antibacterial activity against Streptococcus mutans strain with a mean (11.613 
$\mathrm{mm} \pm$.193) among all tested material by ADT. This result is in accordance with that of Matalon et al, ${ }^{26}$ The large inhibitory zone around the glassionomer and polycarboxylate specimens in ADT might be due to some degree of solubility of its antimicrobial components in aqueous medium. This observation is in agreement with Wheeler et al, ${ }^{27}$ in a similar experiment. Polycarboxylate cement expressed a lower antibacterial effect (mean $9.250 \mathrm{~mm} \pm .957$ ); the material used in this study does not contain fluoride; could be explained by the acidity of the material as it is an acid base material. ${ }^{28}$

In comparison between the chemical and the light cure composite containing fluoride in fresh material, it was found that the chemical cure composite with fluoride had more potent effect, as expressed by halo zone, (mean $7.6 \mathrm{~mm} \pm .2$ ) than the light cure composite containing fluoride with a (mean $7.2 \mathrm{~mm} \pm .263$ ). This may be due to rapid polymerization by light activation. This could be explained as light curing could prevent the hydrogel formation due to water dryness. So, there was little fluoride recharge and movement of remineralization ions of the antimicrobial component take more time to release in the aqueous medium and also, the surface roughness trapping the bacteria present in the media. The light curing composite harden through a polymerization reaction and have limited ionic activity. This accepted with Ewoldsen and Demke (2001). ${ }^{28}$

It is impossible to make generalization about whether a light cured or chemical cured composite resin should be used. This is a clinical advantage of the light cured composite resin system which is more easier in manipulation and better in physical properties. This is accepted with Mandall et al,.. ${ }^{29}$

Chemically-cured composite resin without fluoride (mean $4.4 \mathrm{~mm} \pm .141$ ) and light cured composite resin without fluoride (mean $4.425 \mathrm{~mm} \pm .096$ ) did not exhibit antibacterial phenomena to other composite resin with fluoride as in groups A and B. This put much interest of the role of presence of fluoride in the adhesive material.

\section{CONCLUSIONS}

This in vitro study showed that:

1. The use of Glass ionomer cement (Vivaglass CEM PL) and Polycarboxylate cement (Alpha-Dent) for cementation of orthodontic bands and a chemically-cured composite resin with fluoride (Reliance Orthodontic Prod Inc.) for bonding orthodontic brackets reduced early activity of S. mutans bacterial strain . 
2. Also, fluoride content in the orthodontic adhesives and cements had a greater effect in reducing early activity of S. mutans.

The findings suggest that not all dental adhesives and cements are equally having antibacterial property. Thus, prudent selection of bonding and cementing materials may be used as a means to diminish enamel demineralization during orthodontic therapy.

\section{ACKNOWLEDGEMENTS}

The authors are grateful to Dr. Mohammed A Shalaby, Professor of Virology and Immunology Department, Faculty of Veterinary, Cairo University, for his guidance in giving all facilities in his department, and to Dr Ebraheem El-Sabagh, Lecturer, Virology and Immunology Department, Faculty of Veterinary, Cairo University, for precious help in laboratory work. Also, to Dr. Mahmoud Essam Hatem, Professor and head, Microbiology Department, Faculty of Veterinary, Cairo University, for supplying us with the $\mathrm{S}$ mutans bacteria strain.

\section{REFERENCES}

1. Ogaard B, Rolla G, Arends J. Orthodontic appliances and enamel demineralization. Part 1. Lesion development. Am J Orthod Dentofacial Orthop 1988;94:68-73.

2. Noyes H. Dental caries and the orthodontic patient. J Am Dent Asso 1937;24:1243-54.

3. Chatterjee R, Kleinberg I. Effect of orthodontic band placement on the chemical composition of human incisor tooth plaque. Arch Oral Biol 1979;24:97-100.

4. Gwinnett AJ, Ceen RF. Plaque distribution on bonded brackets: a scanning microscope study. Am J Orthod 1979;75:667-77.

5. Boyd RL. Longitudinal evaluation of a system for self-monitor- ing plaque control effectiveness in orthodontic patients. J Clin Periodontol 1983; 10:380-8.

6. Ogaard B. Prevalence of white spot lesions in 19-year-olds: a study on untreated and orthodontically treated persons 5 years after treatment. Am J Orthod Dentofacial Orthop 1989; 96:423-7. 
7. Corbett JA, Brown LR, Keene HJ, Horton IM. Comparison of Streptococcus mutans concentrations in non-banded and banded orthodontic patients. J Dent Res. 1981; 60:1936-42.

8. Øgaard B, Larsson E, Henriksson T, Birkhed D, Bishara S. Effects of combined application of antimicrobial and fluoride varnishes in orthodontic patients. Am J Orthod Dentofacial Orthop 2001;120:28-35.

9. Buyukyilmaz T, Øgaard B. Caries preventive effects of fluoride- releasing materials. Adv Dent Res. 1995; 9:377-83.

10. Karaman A, Uysal T. Effectiveness of a hydrophilic primer when different antimicrobial agents are mixed. Angle Orthod. 2004;74:414-19.

11. Kawashima M, Nakatsuka K, Okada K, Yamauchi J. Character- istics of a new antibacterial and fluoride releasing bonding system. J Dent Res. 2002; 81:A-241. [abstract 1850].

12. Matasa C G. Microbial attack of orthodontic adhesives. Am J Orthod and Dentofacial Orthop 1995; 108 : 132 - 41.

13. Fox N A. Fluoride release from orthodontic bonding materials. An in vitro study. British Journal of Orthodontics 1990; 17 : 293 - 98.

14. Badawi H, Evans R D, Wilson M, Ready D, Noar J H, Pratten J. The effect of orthodontic bonding materials on dental plaque accumulation and composition in vitro. Biomaterials 2003; $24: 3345$ - 3350

15. Banks P A, Burn A, O'Brien K. A clinical evaluation of the effectiveness of including fluoride into an orthodontic bonding adhesive. European Journal of Orthodontics 1997; 19 : 391- 95.

16. Hallgren A, Oliveby A, Twetman S. Fluoride concentration in plaque adjacent to orthodontic appliances retained with glass ionomer cement. Caries Research 1993; 27 : 51- 54.

17. Dahl B L, 1978 Antibacterial effect of two luting cements on prepared dentin in vitro and vivo. Acta Odontol Scand 36: 363-69.

18. Skold-Larsson K, Borgstrom MK, Twetman S. Effect of an antibacterial varnish on lactic acid production in plaque adjacent to fixed orthodontic appliances. Clin Oral Invest 2001; 5:118-21.

19. Madlena M, Vitalyos G, Marton S, Nagy G. Effect of chlorhexidine varnish on bacterial levels in plaque and saliva during orthodontic treatment. J Clin Dent 2000; 11:42-6. 
Egyptian

Orthodontic Journal

20. Wright AB, Lee RT, Lynch E, Young KA. Clinical and microbiologic evaluation of a resin modified glass ionomer cement for orthodontic bonding. Am J Orthod Dentofac Orthop 1996; 110: 469-75

21. Loesche W J. Microbiology of dental decay and periodontal disease. Barron's medical microbiology. $4^{\text {th }}$ ed (1996).

22. Kindelan JD. In vitro measurement of enamel demineralization in the assessment of fluoride leaching orthodontic bonding agents. Br J Orthod 1996; 23: 343-49.

23. Marcusson A, Norevall L I, Persson M. White spot reduction when using glass ionomer cement for bonding in orthodontics: a longitudinal and comparative study. Eur J Orthod 1997; 19:233-42.

24. Millett DT, Nunn JH, Welbury RR, Gordon PH. Decalcifi- cation in relation to brackets bonded with glass ionomer cement or a resin adhesive. Angle Orthod 1999; 69: 65-70.

25. Millett DT, McCabe JF: Orthodontic bonding with glass ionomer cement: a review. Eur J Orthod 1996; 18: 385-99.

26. Matalon Sh, Slutzky H, and Weiss E I. Antibacterial properties of 4 orthodontic cements. Am J Orthod Dentofacial Orthop 2005;127:56 - 63).

27. Wheeler AW, Foley TF, and Mamandras A: Comparison of fluoride protocols for in-vitro testing of 3 orthodontic adhesives. Am J Orthod Dentofac Orthop 2002; 121:301-9.

28. Ewolsen N and Demke RS : A review of orthodontic cements and adhesives. Am J Ortho dentofacial Orthop 2001; 120: 45-48.

29. Mandall N A, Millett DT, Matick CR, Hickman J, Worthington HV, and Macfarlane TV. Orthodontic adhesives. A system review. Am J Orthod Dentofac Orthop 2002; 9: 205-10. 